IUCrJ

ISSN 2052-2525

BIOLOGY|MEDICINE

Received 14 February 2018

Accepted 4 June 2018

Edited by J. L. Smith, University of Michigan, USA

Keywords: Mycobacterium tuberculosis; methionyl-tRNA synthetase; crystal structure; induced fit; antituberculosis drugs.

PDB references: Mycobacterium tuberculosis MetRS, 5xgq; complex with Met-AMP, 5xet

Supporting information: this article has supporting information at www.iucrj.org

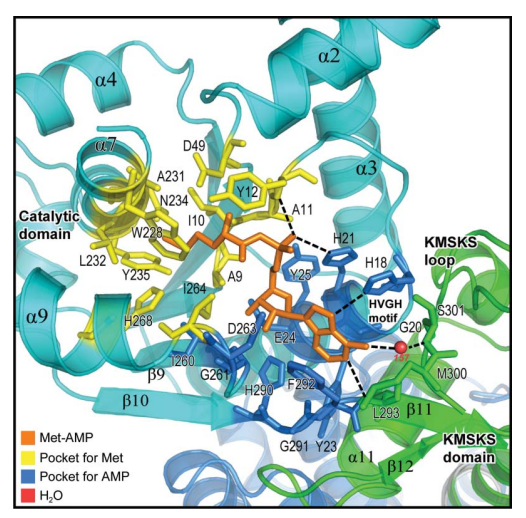

OPEN $\odot$ ACCESS

\section{Structural characterization of free-state and product-state Mycobacterium tuberculosis methionyl-tRNA synthetase reveals an induced-fit ligand-recognition mechanism}

\author{
Wei Wang, ${ }^{\text {a Bo Qin, }}{ }^{\text {J Justyna Aleksandra Wojdyla, }}{ }^{\mathrm{b}}$ Meitian Wang, ${ }^{\mathrm{b}}$ Xiaopan Gao ${ }^{\mathrm{a} *}$ \\ and Sheng $\mathrm{Cui}^{\mathrm{a} *}$
}

${ }^{\mathrm{a}} \mathrm{MOH}$ Key Laboratory of Systems Biology of Pathogens, Institute of Pathogen Biology, Chinese Academy of Medical Science, No. 9 Dong Dan San Tiao, Dong Cheng Qu, Beijing 100730, People's Republic of China, and ${ }^{\mathbf{b}}$ Paul Scherrer Institute, Swiss Light Source, CH-5232 Villigen, Switzerland. *Correspondence e-mail: panda888@ipbcams.ac.cn, cui.sheng@ipb.pumc.edu.cn

Mycobacterium tuberculosis (MTB) caused 10.4 million cases of tuberculosis and 1.7 million deaths in 2016. The incidence of multidrug-resistant and extensively drug-resistant MTB is becoming an increasing threat to public health and the development of novel anti-MTB drugs is urgently needed. MethionyltRNA synthetase (MetRS) is considered to be a valuable drug target. However, structural characterization of $M$. tuberculosis MetRS (MtMetRS) was lacking for decades, thus hampering drug design. Here, two high-resolution crystal structures of MtMetRS are reported: the free-state structure (apo form; $1.9 \AA$ resolution) and a structure with the intermediate product methionyl-adenylate (Met-AMP) bound ( $2.4 \AA$ resolution). It was found that free-state MtMetRS adopts a previously unseen conformation that has never been observed in other MetRS homologues. The pockets for methionine and AMP are not formed in free-state MtMetRS, suggesting that it is in a nonproductive conformation. Combining these findings suggests that MtMetRS employs an induced-fit mechanism in ligand binding. By comparison with the structure of human cytosolic MetRS, additional pockets specific to MtMetRS that could be used for anti-MTB drug design were located.

\section{Introduction}

Mycobacterium tuberculosis (MTB) infection remains a public health challenge owing to its high morbidity and mortality rates. In 2016, the World Health Organization (WHO) reported that 10.4 million people developed tuberculosis and 1.7 million died from the disease (Chetty et al., 2017). Moreover, drug-resistant MTB infections are becoming an increasing threat (Bastos et al., 2014; Hong-min \& Xiao-Hong, 2015). Multidrug-resistant TB (MDR-TB) and extensively drug-resistant TB (XDR-TB) infections have been reported worldwide. Drug-resistant TB often compromises the current anti-TB therapy protocols. Therefore, finding novel drug targets and the development of new drugs against MTB are urgently needed.

Aminoacyl-tRNA synthetases (AARSs) play essential roles in protein synthesis. The 20 essential AARSs are housekeeping enzymes that are present in most organisms. Some microorganisms which do not encode AsnRS and/or GlnRS depend on indirect pathways to synthesize a complete set of aminoacyl-tRNAs (Li et al., 2015). AARSs are considered to be appealing antimicrobial drug targets and therefore have attracted attention in the past two decades (Gadakh \& Van Aerschot, 2012). AARSs catalyze the transfer of amino acids 
to their cognate tRNAs, yielding aminoacyl-tRNAs, which are subsequently used in protein synthesis (Gadakh \& Van Aerschot, 2012). The charging of tRNA is a two-step reaction. Firstly, AARSs recognize a specific amino acid in the presence of ATP and the amino acid is adenylated, resulting in the intermediate product aminoacyl-AMP and pyrophosphate $\left(\mathrm{PP}_{\mathrm{i}}\right)$. Secondly, the amino acid is charged to the acceptor arm of the cognate tRNA and AMP is released (Ibba \& Söll, 2000). AARSs are divided into two major classes, I and II, based on the architecture of the catalytic domain. Class I AARSs are characterized by the presence of two consensus motifs, HIGH and KMSKS, and a catalytic domain exhibiting a canonical Rossmann fold. Class I members bind to the minor groove of the tRNA acceptor stem and transfer the amino acids to the 2 '-hydroxy group of the terminal ribose of tRNA. Based on sequence and structural features, class I AARSs can be further divided into subclasses a, b and c (Deniziak \& Barciszewski, 2001; Ibba \& Söll, 2000; Vondenhoff \& Van Aerschot, 2011; Ingvarsson et al., 2009).

Methionyl-tRNA synthetase (MetRS) is a member of subclass Ia. MetRS is unique among the essential AARSs because it not only charges methionine to tRNA for elongation of translation, but also charges initiator tRNA to initialize translation (Deniziak \& Barciszewski, 2001). Structural studies of MetRSs from various organisms have identified remarkable differences which allow the design of inhibitors that specifically target protein biosynthesis in bacterial pathogens without affecting host-cell activities (Vondenhoff \& Van Aerschot, 2011). MetRS is considered to be one of the most promising drug targets in combatting bacterial pathogens, including MTB.

Inhibitors of MetRS from Staphylococcus aureus (Green et al., 2009; Critchley \& Ochsner, 2008; Critchley et al., 2005; Ochsner et al., 2007) and Clostridium difficile (Critchley et al., 2009; Ochsner et al., 2009) exhibit potent antibacterial activities and are currently in clinical or preclinical trials. However, no inhibitors targeting MtMetRS have been identified to date. A collection of MetRS structures from Aquifex aeolicus, Thermus thermophilus, Pyrococcus abyssi, Escherichia coli and Mycobacterium smegmatis (Nakanishi et al., 2005; Sugiura et al., 2000; Crepin et al., 2004; Mechulam et al., 1999; Ingvarsson \& Unge, 2010) have been determined. Very recently, the structure of M. tuberculosis MetRS (MtMetRS), which shares similar structural characteristics with MetRS from M. smegmatis, was reported in complex with the intermediate methionyl-adenylate (Barros-Álvarez et al., 2018). A comparative analysis of the unliganded structure (apo form or free-state enzyme; F-state) and structures complexed either with the intermediate product, analogues or inhibitors (denoted the product state; P-state) revealed conformation changes induced by the ligands. Earlier this year, the crystal structure of human cytosolic MetRS was released (PDB entry 5gl7; H. Y. Cho, H. J. Lee \& B. S. Kang, unpublished work), providing valuable structural insights into the development of pathogen-specific MetRS inhibitors.

In this study, we successfully purified recombinantly produced MtMetRS. We characterized its enzymatic activity and determined high-resolution crystal structures of F-state MtMetRS and P-state MtMetRS in complex with the intermediate product methionyl-adenylate (Met-AMP). The F-state MtMetRS crystal structure has a nonproductive activesite conformation that has not previously been observed in other MetRS homologues. Binding of the Met-AMP intermediate restores the active-site conformation, indicating that MtMetRS adopts an induced-fit mechanism in ligand binding. Our findings provide an important structural insight that is necessary for the successful design of inhibitors targeting MtMetRS.

\section{Materials and methods}

\subsection{Cloning, protein expression and purification}

The cDNA encoding the gene for MtMetRS (GenBank accession No. CP009480.1) was amplified by polymerase chain reaction (PCR) using the genomic DNA of the M. tuberculosis H37RV strain as the template. A coding sequence for a $6 \times$ His tag was added immediately upstream of the coding sequence for MtMetRS in the upstream primer for the PCR reaction. The PCR product was then inserted between the NcoI and Xhol sites of the pQE-60 vector (Qiagen) to express MtMetRS with double $6 \times$ His tags at the $\mathrm{N}$ - and C-termini, denoted dh-MtMetRS. Next, we introduced a stop codon at the $3^{\prime}$-end of the coding sequence of MtMetRS in a PCR reaction, yielding a plasmid expressing MtMetRS with a single N-terminal $6 \times$ His tag, denoted MtMetRS. Dh-MtMetRS was only used for structure determination of the apo form, while the singly His-tagged protein was used in all other experiments. Plasmids expressing mutants of MtMetRS were prepared using site-directed mutagenesis (KOD-Plus Mutagenesis Kit, TOYOBO) following the manufacturer's instructions. The sequences of the resulting plasmids were verified by DNA sequencing before transformation into E. coli C3016 competent cells (NEB). The bacterial culture was grown in lysogeny broth at $37^{\circ} \mathrm{C}$ to an $\mathrm{OD}_{600}$ of 0.8 . The expression of recombinant MtMetRS was induced by adding $1 \mathrm{~m} M$ isopropyl $\beta$-D-1-thiogalactopyranoside (IPTG), and the bacteria were cultured for a further $10 \mathrm{~h}$ at $28^{\circ} \mathrm{C}$. The cells were then harvested by centrifugation at $4500 \mathrm{rev} \mathrm{min}^{-1}$ for $25 \mathrm{~min}$. The cell pellet was resuspended in lysis buffer consisting of $50 \mathrm{~m} M$ Tris- $\mathrm{HCl} \mathrm{pH} 8.0,200 \mathrm{~m} M \mathrm{NaCl}, 10 \mathrm{~m} M$ $\beta$-mercaptoethanol, $1 \mathrm{~m} M$ PMSF , $10 \mathrm{~m} M$ imidazole. The cells were disrupted by ultrasonication on ice. The lysate was clarified by centrifugation at $13000 \mathrm{rev} \min ^{-1}$ for $1 \mathrm{~h}$. The supernatant was mixed with $2 \mathrm{ml} \mathrm{Ni-NTA}$ resin (Qiagen) and incubated on ice for $1 \mathrm{~h}$ to allow protein binding. The resin was washed thoroughly using lysis buffer before elution with ten column volumes of elution buffer containing $350 \mathrm{mM}$ imidazole. The eluted protein was finally purified using a Superdex 200 column (GE Healthcare) pre-equilibrated with gel-filtration buffer consisting of $20 \mathrm{~m} M$ HEPES $\mathrm{pH}$ 7.0, $100 \mathrm{~m} M \mathrm{NaCl}, 1 \mathrm{~m} M$ DTT. The purified MtMetRS was finally concentrated to $8 \mathrm{mg} \mathrm{ml}^{-1}$ before crystallization trials. 
Table 1

Crystallization conditions, data-collection and refinement statistics and model geometry.

Values in parentheses are for the outer shell.

\begin{tabular}{|c|c|c|}
\hline & MtMetRS & MtMetRS-Met-AMP \\
\hline Crystallization conditions & $\begin{array}{l}0.1 M \text { calcium acetate, } \\
0.1 M \text { sodium } \\
\text { cacodylate, } \mathrm{pH} 5.9 \\
16 \% \text { PEG } 8000\end{array}$ & $\begin{array}{l}0.2 M \text { lithium sulfate } \\
\text { monohydrate, } \\
19 \% \text { PEG } 3350, \\
\text { bis-tris pH } 6.9\end{array}$ \\
\hline Ligand & - & Met-AMP \\
\hline \multicolumn{3}{|l|}{ Data collection } \\
\hline Wavelength (̊̊) & 1.0000 & 0.97776 \\
\hline Beamline & X06DA & BL18U \\
\hline Synchrotron & SLS & SSRF \\
\hline Resolution range $(\AA ̊)$ & $48.69-1.90$ & $37.42-2.38$ \\
\hline No. of reflections & 287337 & 104237 \\
\hline No. of unique reflections & 158725 & 49191 \\
\hline$\langle I / \sigma(I)\rangle$ & $6.49(0.89)$ & $4.77(1.15)$ \\
\hline Completeness (\%) & $96.77(92.13)$ & $99.54(99.83)$ \\
\hline Crystal mosaicity $\left(^{\circ}\right)$ & 0.209 & 0.219 \\
\hline$R_{\text {meas }}(\%)$ & $13.1(108.3)$ & $20.4(92.1)$ \\
\hline Wilson $B$ factor $\left(\AA^{2}\right)$ & 23.59 & 31.33 \\
\hline Space group & $P 1$ & $R 3: H$ \\
\hline $\begin{array}{l}\text { No. of molecules in } \\
\text { asymmetric unit }\end{array}$ & 2 & 1 \\
\hline$a, b, c(\AA)$ & $49.98,72.73,78.38$ & $198.02,198.02,39.16$ \\
\hline$\alpha, \beta, \gamma\left({ }^{\circ}\right)$ & $98.90,90.05,98.47$ & $90.00,90.00,120.00$ \\
\hline \multicolumn{3}{|l|}{ Refinement } \\
\hline$R_{\text {work }}$ & 0.20 & 0.21 \\
\hline$R_{\text {free }}(5 \%$ test set $)$ & 0.24 & 0.25 \\
\hline \multicolumn{3}{|l|}{ No. of atoms } \\
\hline Non-H atoms & 9079 & 4205 \\
\hline Protein & 7916 & 3995 \\
\hline Ligand & 0 & 40 \\
\hline Solvent & 1163 & 170 \\
\hline \multicolumn{3}{|l|}{ Average $B$ factors $\left(\AA^{2}\right)$} \\
\hline Overall & 30.21 & 41.06 \\
\hline Protein & 28.98 & 41.17 \\
\hline Ligand & - & 45.88 \\
\hline Solvent & 38.57 & 34.01 \\
\hline \multicolumn{3}{|l|}{ R.m.s.d.s and stereochemistry } \\
\hline R.m.s.d., bonds ( & 0.0033 & 0.005 \\
\hline R.m.s.d., angles $\left({ }^{\circ}\right)$ & 0.60 & 0.642 \\
\hline \multicolumn{3}{|c|}{ Ramachandran plot, residues (\%) } \\
\hline Favoured region & 97.14 & 97.60 \\
\hline Allowed region & 2.86 & 2.40 \\
\hline Outliers & 0 & 0 \\
\hline \multicolumn{3}{|c|}{ Ligand fit with experimental data } \\
\hline RSCC & - & 0.95 \\
\hline RSR & - & 0.11 \\
\hline \multicolumn{3}{|l|}{ Validation } \\
\hline MolProbity score & $1.46,96$ th percentile & $1.52,99$ th percentile \\
\hline Clashscore, all atoms & $5.4,96$ th percentile & $7.93,97$ th percentile \\
\hline PDB entry & $5 \times g q$ & 5 xet \\
\hline
\end{tabular}

\subsection{Crystallization and structure determination}

Unliganded MtMetRS was crystallized by mixing $1 \mu \mathrm{l}$ dh-MtMetRS protein with $1 \mu \mathrm{l}$ reservoir buffer consisting of $0.1 M$ calcium acetate, $0.1 M$ sodium cacodylate $\mathrm{pH} 5.9,16 \%$ PEG 8000 in a hanging-drop vapour-diffusion system at $22^{\circ} \mathrm{C}$. The MtMetRS-Met-AMP complex was assembled by mixing MtMetRS with a single N-terminal His tag at $8 \mathrm{mg} \mathrm{ml}^{-1}$ with $2.5 \mathrm{~m} M$ ATP, $2.5 \mathrm{~m} M$ L-methionine, $5 \mathrm{~m} M \mathrm{MgCl}_{2}, 1 \mathrm{~m} M$ DTT. The mixture was incubated on ice for $30-60 \mathrm{~min}$ prior to crystallization. The MtMetRS-Met-AMP complex was crystallized by mixing equal volumes of sample and reservoir buffer consisting of $0.2 M$ lithium sulfate monohydrate, $19 \%$
PEG 3350, bis-tris pH 6.9 in a vapour-diffusion system at $22^{\circ} \mathrm{C}$. Crystal cooling was carried out by soaking crystals in reservoir buffer supplemented with $10 \%$ ethylene glycol for 30-60 s before flash-cooling in liquid nitrogen. X-ray diffraction experiments were performed on beamline BL18U at Shanghai Synchrotron Radiation Facility (SSRF), Shanghai, People's Republic of China, and on beamline X06DA at the Swiss Light Source (SLS), Paul Scherrer Institute, Villigen, Switzerland. Complete data sets were collected and processed with the $X D S$ package (Kabsch, 2010). The crystal structure of unliganded MtMetRS was solved by molecular replacement with Phaser (McCoy, 2007) using the M. smegmatis MetRS structure (PDB entry 2x11; Seiradake et al., 2010) as the search model. The crystal structure of the MtMetRS-Met-AMP complex was solved by molecular replacement using the unliganded MtMetRS structure as the search model. The building of the atomic models was completed manually using Coot v.081 (Emsley et al., 2010) and refinement was performed using PHENIX (Adams et al., 2010). The final models have excellent refinement statistics and stereochemical quality. All figures depicting structures were prepared using $P y M O L$ (http://www.pymol.org). The data-collection, structure-refinement and structure-validation statistics are summarized in Table 1.

\subsection{ATP-PP ${ }_{\mathrm{i}}$ exchange activity assay}

The catalytic activity of MtMetRS was assessed using a methionine-dependent ATP-PP ${ }_{i}$ exchange assay with minor modifications (Jørgensen et al., 2000; Ghosh et al., 1991). Briefly, the reaction mixture $(50 \mu \mathrm{l})$ consisted of $0.025 \mathrm{mCi}$ $\left[\gamma_{-}{ }^{32} \mathrm{P}\right]$-ATP, $50 \mathrm{~m} M$ potassium HEPES $\mathrm{pH} 7.5,4 \mathrm{~m} M$ potassium fluoride, $0.02 \%$ gelatine, $10 \mathrm{~m} M$ magnesium chloride, $80 \mathrm{~m} M$ potassium chloride, $2 \mathrm{~m} M \mathrm{PP}_{\mathrm{i}}$ and $1 \mu M$ MetRS. Kinetic constants for methionine were determined in the presence of $2 \mathrm{~m} M$ ATP using 0.01-5 $\mathrm{m} M$ methionine and for ATP in the presence of $2 \mathrm{~m} M$ methionine using $0.1-10 \mathrm{~m} M$ ATP. The reactions were performed at $25^{\circ} \mathrm{C}$. The relative catalytic activities of the mutants were assayed using reaction mixtures consisting of $0.025 \mathrm{mCi}\left[\gamma^{32} \mathrm{P}\right]$-ATP, $50 \mathrm{mM}$ potassium HEPES $\mathrm{pH}$ 7.5, $4 \mathrm{~m} M$ potassium fluoride, $0.02 \%$ gelatine, $10 \mathrm{~m} M$ magnesium chloride, $80 \mathrm{~m} M$ potassium chloride, $2 \mathrm{~m} M \mathrm{PP}_{\mathrm{i}}$ and $1 \mu M$ MetRS. The mixture was pre-incubated at $37^{\circ} \mathrm{C} . \mathrm{PP}_{\mathrm{i}}$ and gelatine were added to the reaction mixture last. Samples $(1 \mu \mathrm{l})$ were removed from the reaction mixture at different time points within $10 \mathrm{~min}$ and spotted onto a cellulose polyethyleneimine TLC plate. The spots were $1.5 \mathrm{~cm}$ from the bottom of the plate and were spaced by $1 \mathrm{~cm}$. Following development with $1 M$ potassium dihydrogen phosphate at $25^{\circ} \mathrm{C}$ in a glass jar, the plates were visualized and quantified using a Typhoon Trio Variable Mode Imager (GE Healthcare).

\subsection{Fluorescence-based thermal shift (FTS) assay}

The FTS assay is based on the principle that ligand binding increases the thermal stability of proteins (Pantoliano et al., 2001). The FTS assay was performed on a CFX96 Real-Time 


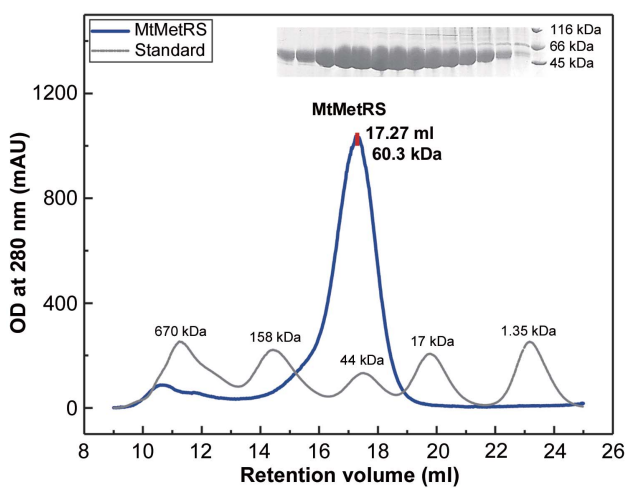

(a)

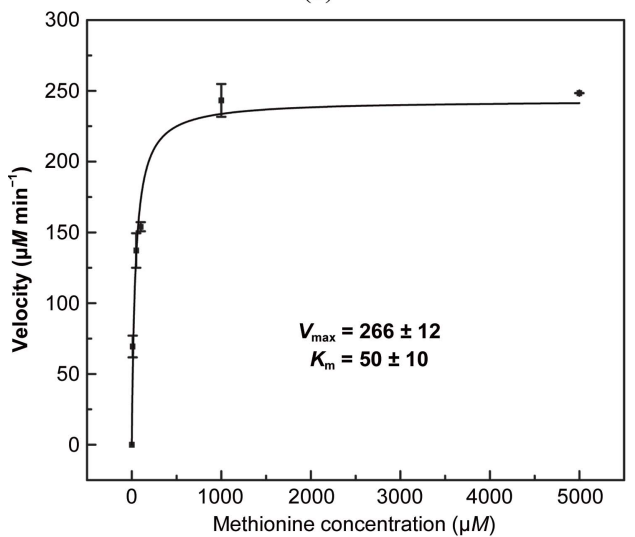

(c) (b)

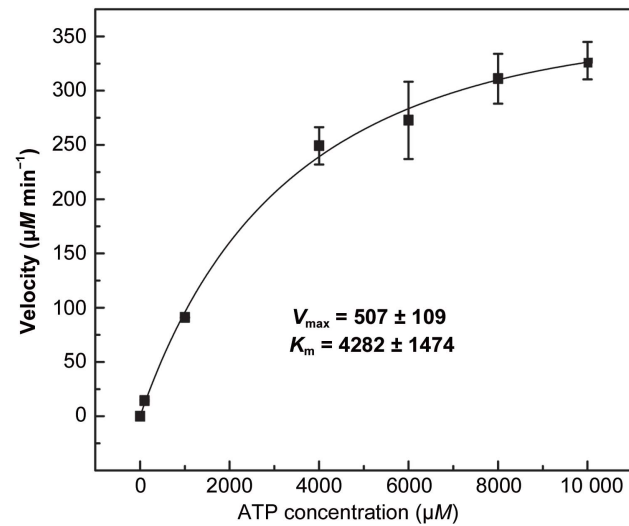

(d)
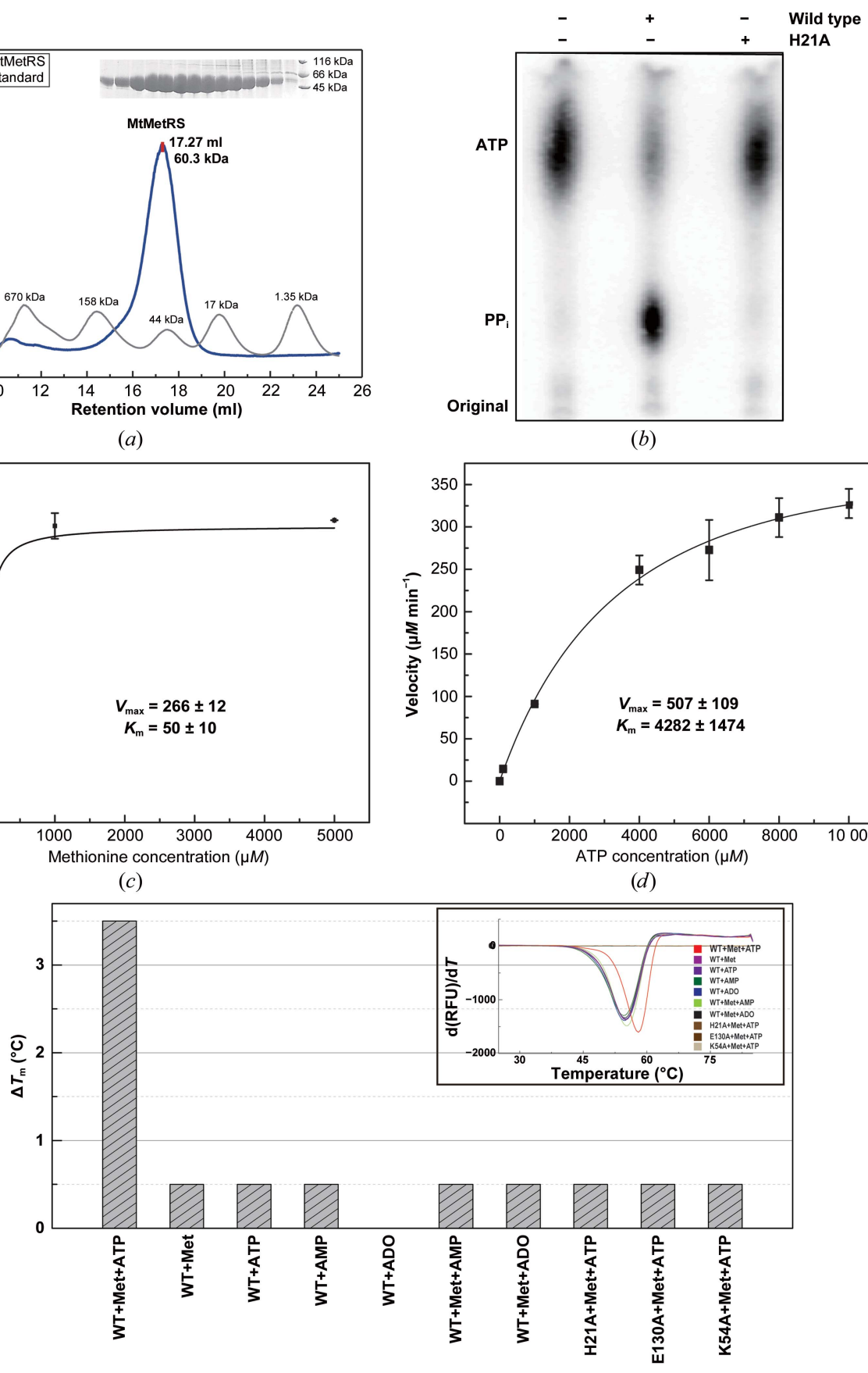

(e)

Figure 1

Biochemical characterization of recombinant MtMetRS. (a) Size-exclusion chromatography of purified recombinant MtMetRS demonstrating that the enzyme is monomeric in solution. The Superdex 200 300/10 GL column was pre-calibrated with the protein standards thyroglobulin $(670 \mathrm{kDa}), \gamma$-globulin $(158 \mathrm{kDa})$, ovalbumin $(44 \mathrm{kDa})$, myoglobin $(17 \mathrm{kDa})$ and vitamin $\mathrm{B}_{12}(1.35 \mathrm{kDa})$. Upper insert, SDS-PAGE analysis of the eluates. $(b)$ ATP-PP ${ }_{i}$ exchange assay showing the catalytic activity of the recombinantly produced MtMetRS. (c) The velocity of ATP-PP $\mathrm{e}_{\mathrm{i}}$ exchange is plotted as a function of methionine concentration. The data were fitted to the Michaelis-Menten equation to calculate $V_{\max }$ and $K_{\mathrm{m}}$ for methionine. $(d)$ The velocity of ATP-PP $\mathrm{P}_{\mathrm{i}}$ exchange is plotted as a function of ATP concentration. The data were fitted to the Michaelis-Menten equation to calculate $V_{\max }$ and $K_{\mathrm{m}}$ for ATP. (e) Thermal shift analysis of protein-ligand interaction. The $T_{\mathrm{m}}$ of the F-state MtMetRS was $55^{\circ} \mathrm{C}$. The histogram displays the melting-temperature $\left(\Delta T_{\mathrm{m}}\right)$ shifts of MtMetRS upon incubation with various ligands: Met, ATP, AMP, ADO (adenosine), Met+AMP, Met+ADO and Met-ATP. The thermal shifts of two catalytically inactive mutants, E130A and $\mathrm{K} 54 \mathrm{~A}$, were also measured. The concentration of the ligands was $200 \mu M$ and the concentration of MtMetRS was $2 \mu M$. In the presence of SYPRO Orange, fluorescence (in relative fluorescence units; RFU) was recorded during heating from 10 to $85^{\circ} \mathrm{C}$ at a rate of $0.5^{\circ} \mathrm{C}$ every $30 \mathrm{~s}$. The upper insert indicates the protein melting temperature $\left(T_{\mathrm{m}}\right)$.
PCR Detection System (Maskell et al., 2015). Each well of a microplate was loaded with $30 \mu \mathrm{l}$ solution consisting of $2 \mu M$ protein, $5 \times$ SYPRO Orange (Invitrogen) and $200 \mu M$ ligand in $50 \mathrm{~m} M$ HEPES $\mathrm{pH} 8,10 \mathrm{~m} M$ $\mathrm{MgCl}_{2}, 80 \mathrm{mM} \mathrm{KCl}$. Additionally, negative controls consisting of $30 \mu \mathrm{l}$ of various ligands and $5 \times$ SYPRO Orange solution in buffer were dispensed onto the microplate. The plates were sealed with sealing foil (Roche) and incubated at $4^{\circ} \mathrm{C}$ for $10 \mathrm{~min}$. The FTS assay was performed on a CFX96 Real-Time PCR Detection System (Bio-Rad) running the following protocol: heating from 10 to $85^{\circ} \mathrm{C}$ with a $30 \mathrm{~s}$ hold time every $0.5^{\circ} \mathrm{C}$. The fluorescence intensity (excitation/ emission at 450-490/560-580 nm) was measured after each cycle. The assays were performed twice for each condition and the melting-temperature $\left(T_{\mathrm{m}}\right)$ values for MtMetRS and mutants were determined by analysing the thermal denaturation curves with the $C F X$ Manager 3.0 software.

\section{Results}

\subsection{Expression and}

characterization of MtMetRS

High-resolution structural characterization of MtMetRS is fundamental to successful targeted structure-based drug design. However, until now the structure of full-length MtMetRS had not been determined owing to difficulties in obtaining soluble protein (Ingvarsson \& Unge, 2010). Therefore, we carried out a systematic optimization of the overexpression conditions, including the selection of an appropriate promoter and bacterial strain, the engineering of suitable affinity tags and optimization of induction, which eventually yielded stable and soluble expression of full-length MtMetRS (see §2). We success- 
fully expressed and purified doubly and singly His-tagged MtMetRS. To minimize the impact of the His tag on the activity of the enzyme, we only used the N-terminally singly His-tagged MtMetRS for biochemical characterization. Next, we performed a size-exclusion chromatography experiment. The molecular mass of N-terminally singly tagged MtMetRS calculated from a size-exclusion chromatography (SEC) experiment, $60.3 \mathrm{kDa}$, closely matched the theoretical molecular mass of $59.3 \mathrm{kDa}$. This result clearly demonstrates that MtMetRS exists as a monomer in solution (Fig. 1a), which is consistent with the oligomeric state of its closest homologue M. smegmatis MetRS (Ingvarsson \& Unge, 2010). Next, we employed an ATP-PP $\mathrm{P}_{\mathrm{i}}$ exchange assay to evaluate the activity of the recombinantly produced MtMetRS. We found that while the wild-type enzyme exhibited evident ATP-PP ${ }_{i}$ exchange activity, the catalytically inactive H21A mutant displayed an activity similar to that of a control without enzyme (Fig. 1b). The $k_{\text {cat }}$ of MtMetRS was calculated as $6 \pm 1 \mathrm{~s}^{-1}$ and the catalytic efficiency $k_{\mathrm{cat}} / K_{\mathrm{m}}$ was $0.0015 \mathrm{~s}^{-1} \mu M^{-1}$ (Figs. $1 c$ and $1 d$ ). Comparison of the enzymekinetic parameters of MtMetRS with those reported for E. coli MetRS (EcMetRS; Ghosh et al., 1991; Table 2) showed that MtMetRS is less efficient than EcMetRS. To assess whether the $\mathrm{N}$-terminal His tag impacts the activity of the recombinant enzyme, we compared our data with the reported activities of MetRSs from various organisms as summarized in Supplementary Table S3. We found that the $K_{\mathrm{m}}^{\text {Met }}$ of MtMetRS was within a reasonable range of reported $K_{\mathrm{m}}$ values. The $K_{\mathrm{m}}^{\mathrm{ATP}}$ of MtMetRS is among the largest reported $K_{\mathrm{m}}$ values. E. coli cells have an average ATP concentration of $1.54 \mathrm{mM}$ (Yaginuma et al., 2014), which is higher than the $K_{\mathrm{m}}^{\mathrm{ATP}}$ of EcMetRS. In contrast, the ATP concentration in M. tuberculosis is approximately $1.0 \mathrm{~m} M$ (James et al., 2000), which is lower than the $K_{\mathrm{m}}^{\mathrm{ATP}}$ of MtMetRS. This analysis may offer an explanation of the slow rate of protein synthesis in MTB and the slow growth rate of this bacterium. It is worth noting that the enzymatic activities of human mitochondrial methionyl-tRNA synthetase were characterized using the N-terminally Histagged enzyme (Green et al., 2009; Spencer et al., 2004). Overall, we believe that the presence of the N-terminal His tag does not affect the activity of recombinantly produced MtMetRS.

To guide the co-crystallization of the MtMetRS-ligand complex, we tested the binding affinities of a selection of ligands using a fluorescence-based thermal shift assay. While F-state MtMetRS (the apo form) exhibited a $T_{\mathrm{m}}$ value of $55^{\circ} \mathrm{C}$, we observed different levels of change in $T_{\mathrm{m}}$ in the presence of various ligands. Among the ligands tested, the largest thermal shift of $3.5^{\circ} \mathrm{C}$ was observed when methionine, ATP and $\mathrm{Mg}^{2+}$ were added to MtMetRS (Fig. 1e), whereas only negligible thermal shifts $\left(0-0.5^{\circ} \mathrm{C}\right)$ were observed for all other ligands. Therefore, a mixture containing MtMetRS, ATP and methionine was selected for co-crystallization. Furthermore, we tested the thermal shifts of two catalytically inactive mutants, K54A and E130A, which were identified based on our structural and mutagenesis studies. Lys54 and Glu130 are involved in the interaction between the catalytic and $\mathrm{CP}$ domains and
Table 2

Pyrophosphate-exchange activity of EcMetRS and MtMetRS.

\begin{tabular}{llccl}
\hline Enzyme & $K_{\mathrm{m}}^{\mathrm{Met}}(\mu M)$ & $K_{\mathrm{m}}^{\mathrm{ATP}}(\mu M)$ & $k_{\mathrm{cat}} \dagger\left(\mathrm{s}^{-1}\right)$ & $K_{\text {cat }} / K_{\mathrm{m}} \ddagger\left(\mathrm{s}^{-1} \mu M^{-1}\right)$ \\
\hline MtMetRS & $50 \pm 11$ & $4282 \pm 1475$ & $6 \pm 1$ & 0.0015 \\
EcMetRS§ & $21 \pm 4$ & $528 \pm 91$ & $74 \pm 8$ & 0.14 \\
\hline
\end{tabular}

$\dagger k_{\text {cat }}$ is the average of the values for methionine and ATP. $\ddagger K_{\mathrm{m}}$ is the $K_{\mathrm{m}}$ for ATP. \& Ghosh et al. (1991).

play an important role in maintaining the catalytically productive conformation of the enzyme (see below). We found that despite the presence of ATP and methionine, both mutants showed a negligible thermal shift $\left(0.5^{\circ} \mathrm{C}\right)$ that was significantly lower than the wild-type shift (Fig. 1e).

\subsection{Structure determination of MtMetRS}

We successfully crystallized both unliganded MtMetRS (denoted F-state MtMetRS) and the enzyme in complex with the intermediate product methionyl-adenylate (Met-AMP; denoted P-state MtMetRS). Dh-MtMetRS with a double His tag was used for the crystallization of the apo-form enzyme, whereas N-terminally His-tagged MtMetRS was used for the crystallization of the Met-AMP-bound enzyme. The crystals of F-state MtMetRS diffracted X-rays to $1.9 \AA$ resolution, belonged to space group $P 1$ and contained two copies of MtMetRS in the asymmetric unit. The crystals of the MtMetRS-Met-AMP complex diffracted X-rays to $2.4 \AA$ resolution and belonged to space group $R 3$, with a single copy of the enzyme in the asymmetric unit. We located 489 residues in chain $A$ and 488 residues in chain $B$ out of a total of 519 amino acids in MtMetRS, whereas 504 residues were found in the Met-AMP-bound structure. In particular, there was no electron density for the entire $\alpha 2$ helix between $\beta 2$ and $\alpha 3$ in the unliganded structure, whereas the $\alpha 2$ region was well ordered in the structure of the MtMetRS-Met-AMP complex. To rule out the possibility that the missing residues in the unliganded structure were a consequence of proteolysis during crystallization, we collected crystals of the unliganded enzyme from the crystallization drops, dissolved the crystals and analyzed the sample by SDS-PAGE. As seen in Supplementary Fig. S1, the protein in the unliganded enzyme crystals migrated similarly to the purified protein. Therefore, we conclude that the residues that are missing in the unliganded MtMetRS structure reflect intrinsic flexibility of the protein and that the binding of Met-AMP stabilizes the catalytic domain. We summarize the data-collection, structurerefinement and validation statistics in Table 1.

\subsection{Overall structure of MtMetRS}

The overall structure of MtMetRS is similar to those previously reported for MetRSs from various species. The catalytic domain of MtMetRS spanning residues 1-115 (the $\mathrm{N}$-terminal segment) and residues 226-292 (the C-terminal segment) has a typical $\alpha / \beta$ Rossmann fold. There are five parallel $\beta$-strands ( $\beta 1-\beta 3$ and $\beta 9-\beta 10)$ surrounded by seven $\alpha$-helices $(\alpha 1-\alpha 4$ and $\alpha 7-\alpha 9)$. The connective polypeptide (CP) domain (residues 116-225) is inserted between the 
$\mathrm{N}$-terminal and C-terminal segments of the catalytic domain. While the $\alpha$-helix-rich subdomain of the CP domain $(\alpha 5-\alpha 6)$ tightly binds the $\alpha 9$ helix and the $\beta 10$ strand of the catalytic domain, the $\beta$-rich subdomain of the $\mathrm{CP}$ domain $(\beta 4-\beta 7)$ wraps into an arched parallel $\beta$-strand covering the top of the active site (Fig. 2a, Supplementary Fig. S2). The tip of the CP domain is located between $\beta 4$ and $\beta 8$, which harbours one 'knuckle' but lacks the metal-coordination site. This structural feature classifies MtMetRS into the MetRS1 subfamily (Deniziak \& Barciszewski, 2001). The KMSKS domain (resi-

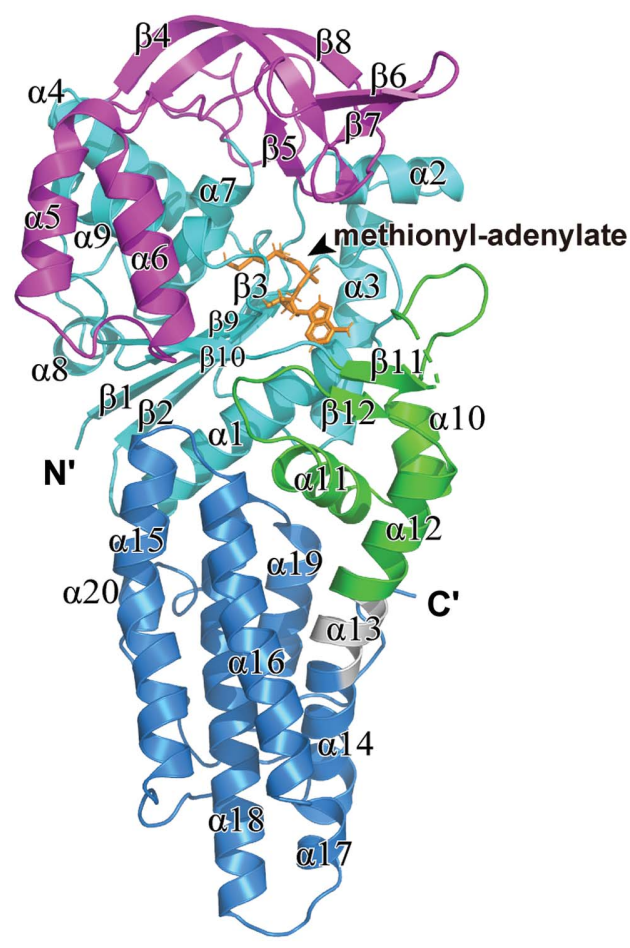

(a)

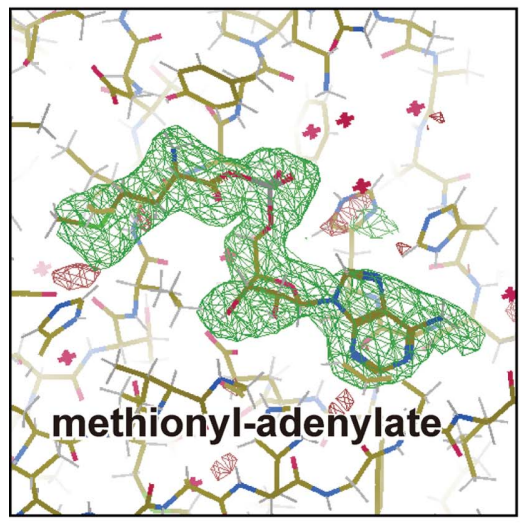

(c)

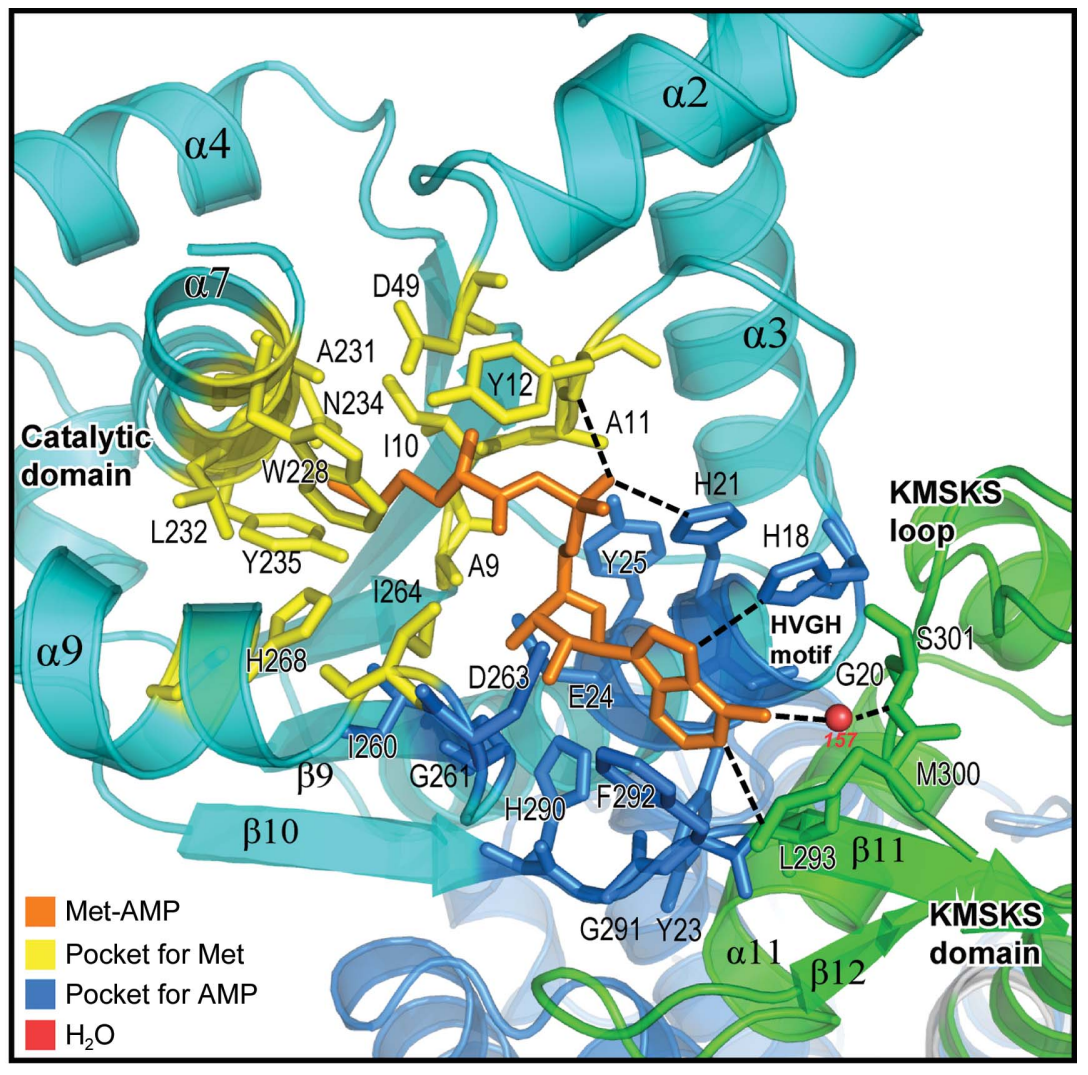

(b)

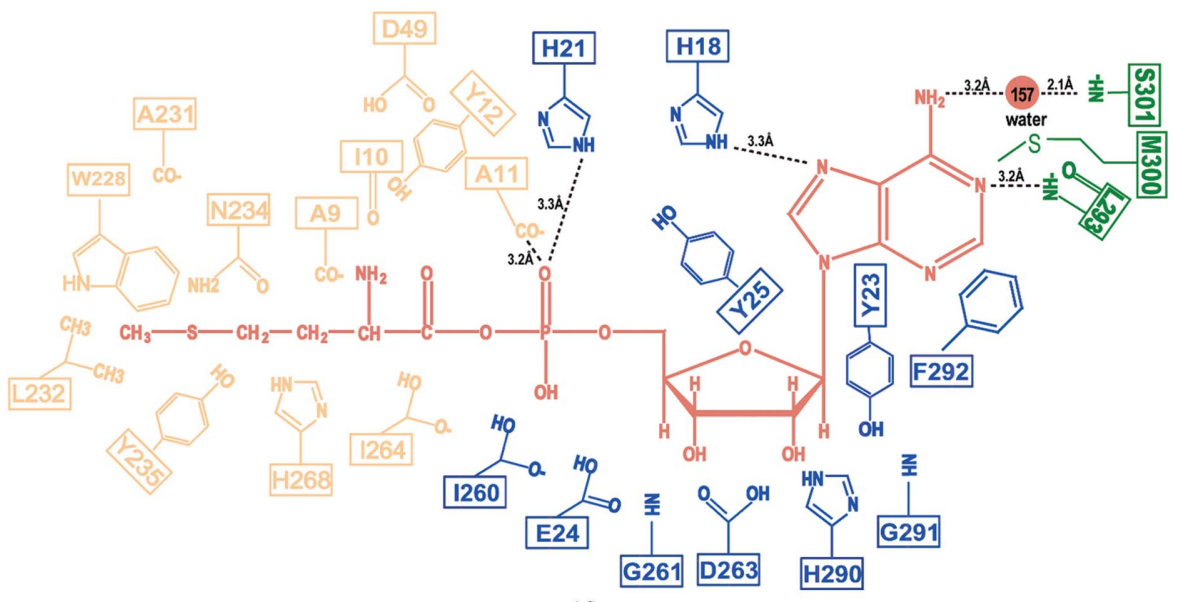

(d)

Figure 2

Overall structure of MtMetRS-Met-AMP and the architecture of the active site. (a) Ribbon model of MtMetRS. The individual domains are differently coloured, with the catalytic domain in cyan, the CP domain in magenta, the KMSKS domain in green and the anticodon domain in blue. The docking of the intermediate product Met-AMP in the active site is shown as a stick model in orange. The $\pi$-helix $\alpha 13$ connecting the KMSKS domain and the anticodon domain is shown in grey. Secondary-structure features of the structure are labelled. $(b)$ A magnified view of the MtMetRS structure showing the details of the active site. Residues making up the substrate-binding pockets are shown as stick models and labelled. The methionine pocket is highlighted in yellow and the AMP pocket is highlighted in blue. Met-AMP is shown as a stick model and coloured orange. Ordered waters mediating the interaction between MtMetRS and Met-AMP are shown as red spheres. Hydrogen bonds between MtMetRS and Met-AMP are indicated by dashed lines. (c) A magnified view of the structure of the active site with a superimposed polder OMIT map for Met-AMP. The map is contoured at $\pm 3 \sigma$ with green and red densities. $(d)$ A two-dimensional diagram of the active site of MtMetRS occupied by Met-AMP. The colour scheme is the same as that in (b). Hydrogen bonds are shown as dashed lines and the bond lengths are indicated. 
dues 293-350) harbouring the signature ${ }_{299} \mathrm{KMSKS}_{303}$ sequence (or KMSKS loop) is also known as the stem-contactfold domain. The KMSKS domain borders the catalytic domain and the anticodon domain. The C-terminal anticodon domain (residues 358-519) is a helix-rich domain comprised of seven antiparallel helices $(\alpha 14-\alpha 20)$. A small $\pi$-helix $(\alpha 13$; residues 351-357) connects the KMSKS domain and the anticodon domain (Supplementary Fig. S2).

We compared the P-state MtMetRS structure with all entries in the Protein Data Bank using the DALI server (Holm \& Laakso, 2016; http://ekhidna2.biocenter.helsinki.fi/ dali/index.html\#tabs-3). The best hit was M. smegmatis MetRS (MsMetRS). Structural comparison between MtMetRS and MsMetRS aligned $499 \mathrm{C}^{\alpha}$ atoms and gave a DALI Z-score of 54.4 and an r.m.s.d. value of $0.8 \AA$. Comparison of the MtMetRS structure with all other MetRS structures resulted in $D A L I Z$-scores ranging from 44.2 to 54.4 (Supplementary Table S1).

\subsection{The active site}

We pre-incubated methionine, ATP and $\mathrm{Mg}^{2+}$ with the MtMetRS protein prior to crystallization trials, which allowed the synthesis of an intermediate product in the first step of tRNA charging: methionyl-adenylate (Met-AMP). As expected, we captured a catalytic component complex with Met-AMP fully occupying the active site, providing atomic insight into the recognition of Met-AMP by MtMetRS. The electron-density map (polder OMIT map; Liebschner et al., 2017) for the intermediate product is very clear, allowing the assignment of all of the Met-AMP atoms (Fig. 2c, Supplementary Fig. S3). The fit between the experimental electron density and Met-AMP in the final model was assessed using the real-space correlation coefficient (RSCC; 0.95) and the real-space $R$ value (RSR; 0.11). These values suggest a suitable fit between the experimental data and the model, and a good agreement between the observed and calculated electron densities for Met-AMP in our structure (Table 1). Our structure reveals that the active site is located on top of the $\alpha 1$ helix and the C-terminal end of the $\beta$-strand plane, and is surrounded by helices $\alpha 2, \alpha 3, \alpha 7$ and $\alpha 9$. The active site can be divided into a binding pocket for methionine and a binding pocket for AMP. The methionine pocket is a deep cavity built up by 12 highly conserved residues, whereas the AMP pocket is a deep groove formed by 15 conserved residues (Figs. $2 b$ and $2 c$ ). While methionine and the ribose and adenosine base parts of Met-AMP are deeply buried, the $\alpha$-phosphate connecting the adenosine and methionine moieties is more exposed to solvent. The O1S atom of the $\alpha$-phosphate is specifically recognized by multiple hydrogen bonds donated by Ala11 and His 21 (Figs. $2 b$ and $2 c$ ). His18 and His21 belong to the highly conserved HIGH motif $\left({ }_{1} \mathrm{HVGH}_{21}\right.$ in MtMetRS), in which the $\mathrm{N}^{\varepsilon 2}$ atom of the His18 side chain forms a hydrogen bond to the N7 atom of the adenosine base (Figs. $2 b$ and $2 c$ ). Therefore, the HVGH motif recognizes both the $\alpha$-phosphate and the adenosine base of Met-AMP. The ribose part of Met-AMP is recognized by several hydrogen-bond interactions. The
Table 3

Residues of MtMetRS recognized by Met-AMP (distance cutoff $3.0 \AA$ ).

\begin{tabular}{lll}
\hline Met-AMP atom & MtMetRS residue & Ordered water \\
\hline O2S & \\
P1 & \\
O1S & Ala11, Tyr12, His21 \\
O1 & Ala11, Tyr12 \\
C9 & Trp228 \\
C $^{\alpha}$ & Ala9, Ile10, Ala264, Trp228 \\
C $^{\beta}$ & Ile10, Trp228 \\
C $^{\gamma}$ & Ile10, Ala231, Leu232, Asn234, Tyr235 \\
S $^{\delta}$ & Ile10, Ale, Tyr12, Asp49 \\
C $^{\varepsilon}$ & Ile264 \\
N2 & Ala11, His21 \\
O & Ala9, Glu24 \\
C5' & Ile264 \\
C4' & Glu24, Gly261, Ile264 \\
C3' & \\
O3 $^{\prime}$ & Gly261, Asp263, Ile264 \\
C2 & His290 \\
O2 $^{\prime}$ & Ala9, Glu24, \\
O4 $^{\prime}$ & His290 \\
C1 & His290, Gly291, Phe292 \\
C4 & His18 \\
N3 & \\
C2 & Gly20 \\
C8 & Gly20, Phe292, Leu293 \\
N7 & Leu293 \\
C5 & & \\
C6 & N1 & Water-157 \\
N6 & & \\
\hline
\end{tabular}

2'-hydroxyl group of ribose accepts two hydrogen bonds from the side chain of Asp263, the backbone NH group of Gly261 and the side chain of Ile264. The $3^{\prime}$-hydroxyl group of ribose accepts a hydrogen bond from the side chain of Glu24. The KMSKS loop (located at the tip of the KMSKS domain) forms the distal end of the methionine pocket. The KMSKS loop specifically recognizes the adenosine base of AMP. The adenine ring forms two hydrogen bonds to the carbonyl $\mathrm{O}$ atom and backbone NH group of Leu293. The aromatic side chain of Phe292 stabilizes the adenosine base via $\pi-\pi$ stacking. Additionally, an ordered water molecule (water-157) bridges hydrogen-bond interactions between the N6 atom of the adenosine base and the NH group of Ser301 (Figs. $2 b$ and 2c). All contacts between MtMetRS and Met-AMP are summarized in Table 3.

We compared the architectures of the active sites of MtMetRS and MsMetRS, demonstrating that the residues that mediate the recognition of Met-AMP are highly conserved (Supplementary Fig. S2). Minor differences include Phe292 in MtMetRS, the counterpart of which in MsMetRS is Trp294. Because Phe292 also has an aromatic side chain, it fulfils the same function of stabilizing the adenosine base of AMP via $\pi$-stacking. A handful of MetRS structures containing MetAMP (or an analogue) are available in the PDB, including those of Trypanosoma brucei MetRS-Met-AMP (TbMetRS; PDB entry 4eg3; Koh et al., 2012), Leishmania major MetRS-Met-AMP-PP (LmMetRS; PDB entry 3kfl; Larson et al., 2011), E. coli MetRS-methionyl sulfamoyl adenosine (EcMetRS; PDB entry 1pfy; Crepin et al., 2003) and A. aeolicus MetRS-methionyl sulfamoyl adenosine (AaMetRS; PDB 
entry 2ct8; Nakanishi et al., 2005). Structural comparison of these MetRS homologues revealed that while the residues recognizing the adenine, phosphate or methionine moieties of Met-AMP remain highly conserved, the residues recognizing the ribose part are more variable (Supplementary Fig. S2).
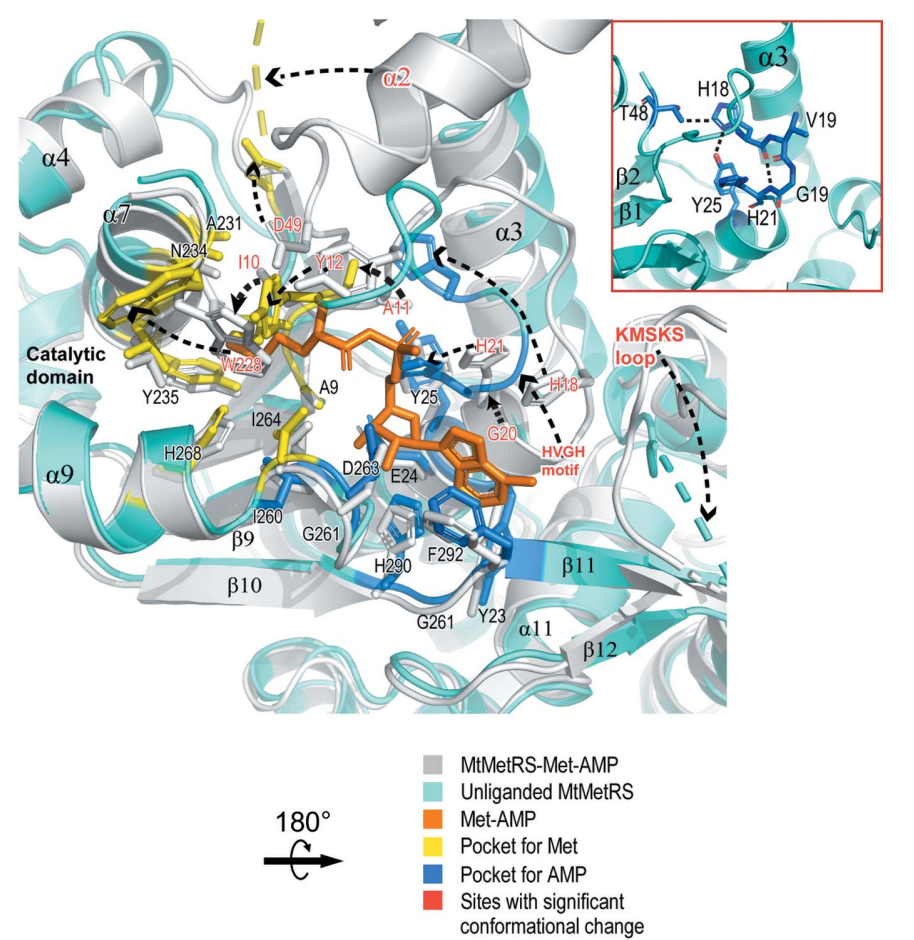

(a)

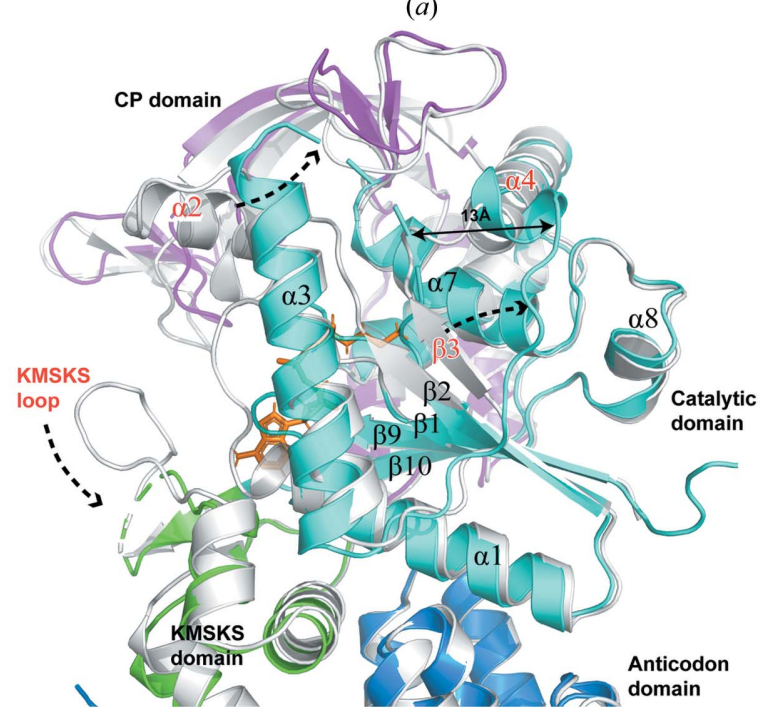

(b)

Figure 3

Conformational changes associated with Met-AMP binding. (a) The structure of the active site of F-state MtMetRS (cyan) is superimposed with the active site of P-state MtMetRS (grey). The residues that make up the substrate-binding pockets are shown as stick models. The methionine pocket is highlighted in yellow and the AMP pocket is highlighted in blue. Met-AMP bound to the P-state enzyme is shown as a stick model (orange). Large structural rearrangements between these two structures are indicated by the dashed arrows with structural elements labelled in red. The inset shows the unusual $\beta$-turn conformation of the HVGH motif in F-state MtMetRS. Secondary-structure elements are labelled. (b) View from the opposite side to that in $(a)$. Large rearrangements include the KMSKS loop, the $\alpha 2$ and $\alpha 4$ helices and the $\beta 3$ strand.
3.5. Extreme changes of the active-site conformation associated with Met-AMP binding

To analyze the conformational changes associated with Met-AMP binding, we compared the structures of F-state and P-state MtMetRS using the pairwise DaliLite server (http:// ekhidna.biocenter.helsinki.fi/dali_lite/start). There were two chains $(A$ and $B)$ in the F-state crystal structure, which are nearly identical. $D A L I$ pairwise structural alignment between chains $A$ and $B$ gave a $Z$-score of 58.3 and an r.m.s.d. value of $0.2 \AA$ for 495 (out of a total of 496) aligned residues. Chain $B$ was used for further structural analysis because of its lower average $B$ factor. To our surprise, the comparison between F-state and P-state MtMerRS resulted in a DALI Z-score of 46.6 with $479 \mathrm{C}^{\alpha}$ atoms aligned and an r.m.s.d. value of $1.8 \AA$. This comparison indicates significant differences between the F-state and P-state structures, which are greater than those between MetRS homologues across species (M. tuberculosis, M. smegmatis, Brucella melitensis etc.). We further compared the individual domains of F-state and P-state MtMetRS, and found that while the r.m.s.d. between the catalytic domains was $2.1 \AA$, the r.m.s.d.s between the other domains were less than $1.6 \AA$ (Supplementary Table S2), suggesting that the largest conformational change occurred within the catalytic domain.

We illustrate in Fig. 3(a) that the residues constituting the active site undergo dramatic conformational rearrangements in the absence of the ligand. The nucleotide-binding loop harbouring the conserved $\mathrm{HVGH}$ motif does not have a general helical conformation; instead, it adopts a rare type II $\beta$-turn conformation (Fig. 3a). Surprisingly, His18 has moved upwards by $8.2 \AA$ ( $\mathrm{C}^{\alpha}$ distance $)$ and its side chain is inserted between the parallel $\beta$-strands $(\beta 1-\beta 3)$ and the $\alpha 3$ helix. The side chain of His 21 tilts towards the $\alpha$-phosphate of Met-AMP. The KMSKS loop forming the distal end of the AMP pocket was completely missing in the F-state enzyme. Ile10 and Tyr12, which constitute the upper wall of the methionine cavity, push into the methionine pocket $\left(\mathrm{C}^{\alpha}\right.$ displacements of 2.6 and $5.1 \AA$ ) and hence the pocket essentially collapses. Moreover, the rearrangement of Tyr12 causes its side chain to clash with the original position of Trp228 (Fig. 3a), inducing movement of the side chain of $\operatorname{Trp} 228$, which constitutes the opposite wall of the cavity, away from the active site.

The large conformational rearrangements of the active site also spread to neighbouring regions (Fig. $3 b$ ). In F-state MtMetRS the entire $\alpha 2$ helix (residues 50-62) connecting $\beta 2$ and $\alpha 3$ disappeared from the electron-density map, suggesting high flexibility of this region. A polypeptide segment (residues 90-101) located on the opposite side of the active site altered the backbone trace completely (Fig. $3 b$ ). Thr95 from this segment underwent the largest displacement. The $\mathrm{C}^{\alpha}$ atom of Thr95 shifted $\sim 13 \AA$ in the F-state structure with respect to its original position in the $\mathrm{P}$-state structure. As a result, the $\beta 3$ strand was separated from the $\beta 2$ strand and the N-terminal part of the $\alpha 4$ helix became distorted.

Collectively, our crystallographic investigations revealed a previously unobserved active site in F-state MtMetRS which 
appears to be nonproductive. We illustrate in Figs. 4(a) and 4(b) that while the active site is well ordered in P-state MtMetRS, the methionine pocket collapses and the AMP pocket shrinks significantly in the F-state enzyme. We used the $C A S T p$ software to calculate the volumes of the active sites in both structures (Dundas et al., 2006). The cavity of the active site has a volume of $2186 \AA^{3}$ in the presence of Met-AMP; in contrast, it reduced to $360 \AA^{3}$ in the absence of the ligand.

\subsection{Mutagenesis study}

To validate our structural findings, we carried out a mutagenesis study. Our structural analyses showed that while the $\alpha 2$ helix was stabilized by the CP domain via two hydrogen bonds (from the Glu130 side-chain $\mathrm{O}^{\varepsilon 2}$ atom to Lys53 $\mathrm{NH}$ and from the Lys54 side-chain $\mathrm{N}^{\zeta}$ atom to the carbonyl group of

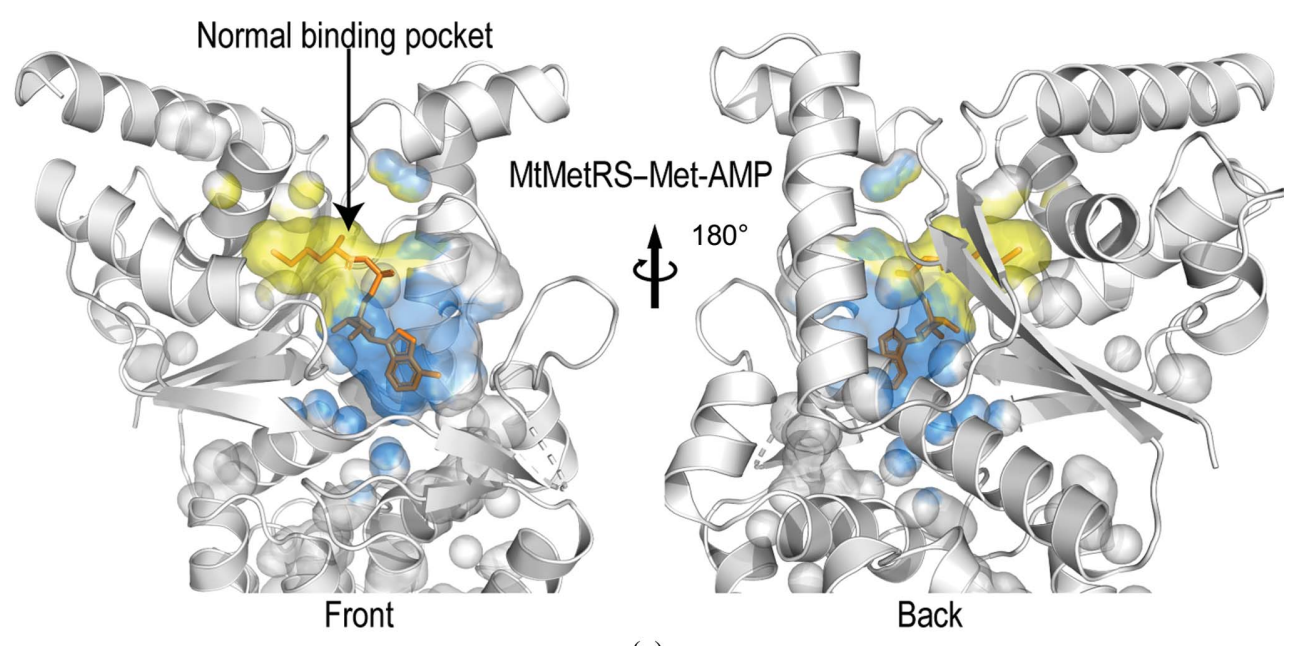

(a)

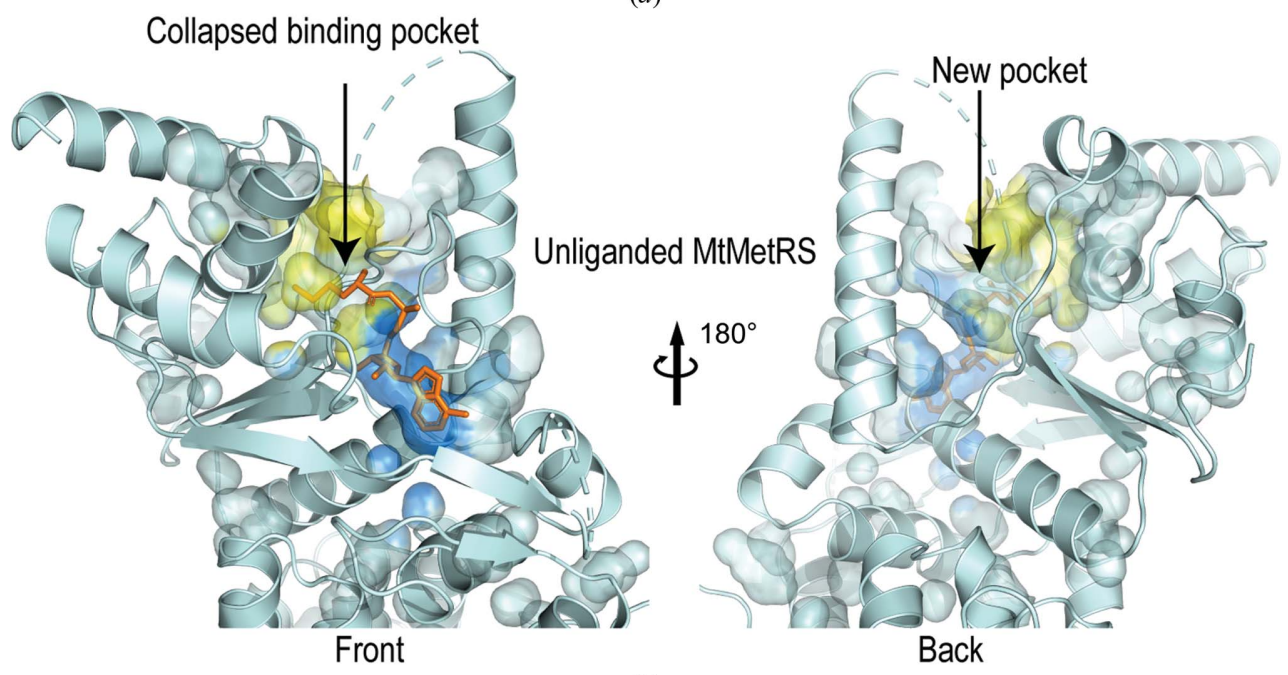

(b)

Figure 4

Shrinkage of the active-site cavity in the absence of Met-ATP. (a) Ribbon model of P-state MtMetRS (grey) with a semitransparent surface for the cavities and pockets. Left, front view; right, rear view. The methionine pocket is shown in yellow and the AMP pocket in blue. Met-AMP is shown as an orange stick model. (b) Ribbon model of F-state MtMetRS (grey) with a semitransparent surface for the cavities and pockets. Left, front view; right, rear view. The methionine pocket is shown in yellow and the AMP pocket is in blue. Met-AMP (orange stick model) is modelled in the active site of the F-state structure by superimposing it with the P-state structure, which demonstrates that the collapsed substrate-binding pockets cannot accommodate Met-AMP.
Arg128; Fig. 5a) in the Met-AMP-bound structure, the corresponding hydrogen bonds were not preserved in the F-state structure and the $\alpha 2$ helix disappeared completely. Using site-directed mutagenesis, we introduced K54A and E130A mutations into MtMetRS to disrupt the hydrogen bond between the $\mathrm{CP}$ domain and the $\alpha 2$ helix. We then measured the ATP-PP $\mathrm{P}_{\mathrm{i}}$ exchange activity of these mutants and found that neither the K54A mutant nor the E130A mutant retained activity (Fig. 5b). The MtMetRS structure shows that Lys54, Glu130 and Arg128 are not in direct contact with Met-AMP; therefore, their essential role in catalysis is more likely to be in the formation of a productive enzyme conformation. This result also suggests that in the absence of ligand the unusual catalytically inactive state.

The conserved aromatic residue Phe292 in MtMetRS is located between the catalytic and KMSKS domains and plays a role in stabilizing the adenosine base of Met-AMP. The corresponding residue in other MetRS homologues varies among phenylalanine, tryptophan and tyrosine. We substituted Phe292 with alanine, histidine and tyrosine, respectively. The F292A mutant lost most of the ATP-PP exchange activity and the F292H mutant lost nearly half of the activity; however, the F292Y mutant showed nearly no loss of activity (Fig. 5). This result suggests that stabilization of the adenosine base is critical for the formation of Met-AMP.

\section{Discussion}

In a structural characterization of EcMetRS, Serre and coworkers reported that the binding of methionine induced the rearrangement of several aromatic residues at the active site (Serre et al., 2001). We observed conformational changes of MtMetRS upon binding by the intermediate product Met-AMP, and the structural rearrangements that occurred in MtMetRS were on a much larger scale. The unusual architecture of the active site of F-state MtMetRS and the large rearrangements of the substratebinding pockets associated with Met-AMP binding, involving a large displacement of the back- 
bone, alteration of the secondary structure and side-chain rocking, have never been observed in other MetRS homologues. Sequence-conservation analysis of different MetRS homologues showed that the $\alpha 2$ and $\alpha 3$ helices of the catalytic core are less conserved than other parts of the protein. As a result, we found that the hydrogen bonds formed between the $\alpha 2$ and $\alpha 3$ helices and the nucleotide-binding loop in the structures of other MetRS homologues (E. coli MetRS, T. thermophilus MetRS, P. abyssi MetRS and human cytoplasmic MetRS) are absent in the MtMetRS structure. The absence of these hydrogen bonds may be responsible for the high flexibility of the catalytic core of MtMetRS in the absence of ligands. The structure of the MtMetRS-Met-AMP complex demonstrates that the ligand and the $\mathrm{CP}$ domain can stabilize the catalytic core and effectively restore the active conformation of the catalytic domain. The unusual F-state structure of MtMetRS observed here reflects the intrinsic flexibility of the catalytic core, which is unique among the characterized MetRSs. This nonproductive conformation probably represents one of the conformations that exist in an ensemble of conformations of MtMetRS.

Crystal packings are nonspecific interactions involving patches on a protein surface with a size that is generally smaller than that of the specific binding interfaces (Carugo \& Argos, 1997). Therefore, crystal packing belongs to the weak protein-protein interactions; it may induce subtle conformational changes to surface loops but rarely alters the folding of a domain core. We analyzed the crystal packing of dhMtMetRS using PISA (http://www.ebi.ac.uk/pdbe/pisa/). The largest packing interface area has a size of $1155 \AA^{2}$ with a $\Delta^{i} G$ of $-2.4 \mathrm{kcal} \mathrm{mol}^{-1}$, and involved 36 residues of one chain and 39 residues of another. This interaction was not recognized as a specific interaction by the software. We next analyzed the packing interactions in detail, revealing two distinct sites. (i) The N-terminal $6 \times$ His tag of one chain occupies a shallow groove located on top of the $\mathrm{CP}$ domain of another chain between the $\alpha$-helix-rich and $\beta$-rich subdomains. The $6 \times$ His tag was clearly visible in the electron-density map and is most likely to play a role in lattice formation (Supplementary Fig.

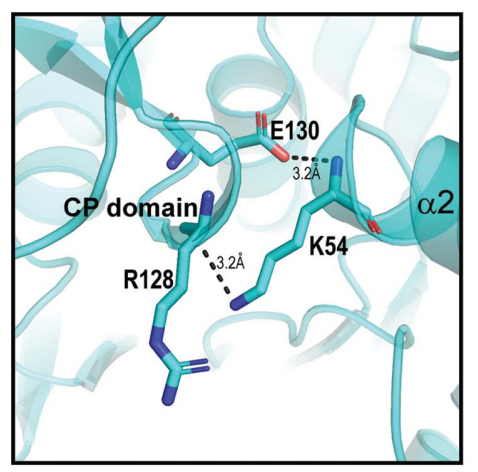

(a)

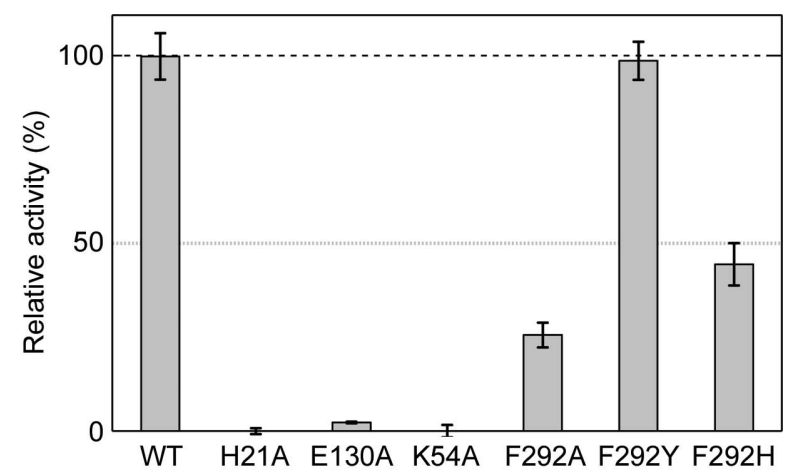

$(b)$
Figure 5

ATP-PP ${ }_{\mathrm{i}}$ exchange assay of various MtMetRS mutants. (a) Hydrogen bonds between the CP and catalytic domains are shown by dashed lines; the bond lengths are indicated. A histogram presentation of the ATP$\mathrm{PP}_{\mathrm{i}}$ exchange activities of a collection of mutants. The catalytic activities of the mutants are expressed as the percentage relative activity compared with wild-type MtMetRS. The result represents triplicate independent measurements with error bars from calculated standard deviations.
S6a). Comparing the F-state and P-state MtMetRS structures, which have different space groups and packing interactions, reveals that the structure of the fold of the $\mathrm{CP}$ domain remains unchanged, suggesting that the packing force was too weak to affect the conformation. (ii) The catalytic domain of dhMtMetRS interacts with the CP domain of another chain (Supplementary Fig. S6b). The polypeptide segment (between the $\alpha 3$ helix and the $\alpha 4$ helix) exhibiting different conformation in apo and liganded structures is located next to the contact site, but this region is not directly involved in interaction. Another crystal-packing interaction involving the catalytic domain is found between the $\alpha 3$ helix and the $\alpha 6$ helix from the CP domain of a nearby chain (Supplementary Figs. S $6 c$ and S $6 d$ ). The HVGH motif adopting the rare $\beta$-turn conformation in the apo structure is not involved in crystal contacts, suggesting that the observed conformation does not result from crystal-packing artifacts. In the P-state structure, the $\alpha 2$ helix (which is disordered in the apo structure) has a much higher $B$ factor $\left(64.8 \AA^{2}\right)$ than the average $B$ factor of the entire chain $\left(41.1 \AA^{2}\right)$, which is unusual for a secondarystructural element. The $\alpha 2$ helix of MtMetRS harbours a unique polyalanine segment (residues 58-62) that is followed by a hydrophobic loop between the $\alpha 2$ and $\alpha 3$ helices; thus, this region forms a large hydrophobic patch on the molecular surface. Therefore, our analysis shows that the structure of the $\alpha 2$ region is intrinsically unstable. It is possible that in the absence of the ligand the folding of this region cannot be maintained and it may become disordered, as observed in the F-state structure. Collectively, our analyses demonstrate that it is unlikely that crystal-packing interactions resulted in the drastic changes at the active site of the F-state enzyme.

In April 2018, Barros-Álvarez and coworkers reported a crystal structure of MtMetRS complexed with the intermediate product Met-AMP (PDB entry 6ax8; Barros-Álvarez et al., 2018). It has a lower resolution than PDB entry 5xet; thus, our crystallographic data may provide further structural details. In particular, the clear electron-density map of PDB entry 5xet allowed us to build the intermediate product with high accuracy (Fig. 2c). The Met-AMP shows a very good fit to the data (RSCC 0.95, RSR 0.11); however, the geometry of the ligand appears to be unusual. In contrast, the Met-AMP in PDB entry $6 \mathrm{ax} 8 \mathrm{had}$ a poorer fit to the data (RSCC 0.84, RSR 0.24) despite exhibiting nearly ideal geometry. To understand this contradiction, we compared the two structures (Supplementary Fig. S7). We found that the aromatic side chain of Phe292 is nearly parallel to the adenine base of Met-AMP in PDB entry 5xet, indicating that Phe292 interacts with the adenine via $\pi-\pi$ stacking. In contrast, the side chain of Phe292 is almost normal 
to the adenine plane of Met-AMP in PDB entry 6ax8, suggesting that the $\pi$-stacking does not form. This observation suggests that while the adenine base of Met-AMP is recognized by Phe292 in PDB entry 5xet, it is not fully bound in PDB entry 6ax8. Superimposition of the two Met-AMP structures demonstrates that the orientations of their adenine bases account for the largest structural deviation. Therefore, the conformation of Met-AMP observed in PDB entry 5xet might represent an unusual state different from that in PDB entry 6ax8. The conformation of Met-AMP in PDB entry 5xet may represent a metastable state, which coincides with the unusual ligand geometry.

Koh and coworkers reported eight structures of T. brucei MetRS (TbMetRS) complexed with various ligands (Koh et al., 2012). They revealed a surprising structural plasticity of TbMetRS which allows the enzyme to adopt many different conformations depending on the bound inhibitor. Their analyses suggested that in F-state MetRS the ligand-binding pockets, including the expanded methionine pocket (EMP) and the auxiliary pocket (AP), were pre-formed and open for ligand access. Therefore, they proposed a conformational selection mechanism rather than induced fit. In sharp contrast, we found that F-state MtMetRS is characterized by a completely collapsed methionine pocket, a nonexistent AP (Supplementary Fig. S4) and a severely reduced groove for adenosine. The binding of Met-AMP induced a conformational change in the nucleotide-binding loop and the active site of MtMetRS was restored to a normal P-state conformation that has no significant differences from the P-state conformation observed in other MetRS homologues (Fig. 6). Combining these analyses suggests that MtMetRS adopts an induced-fit mechanism rather than conformation selection in ligand binding.

Induced-fit and conformation selection are two generally accepted mechanisms for ligand recognition (Vogt \& Di Cera,
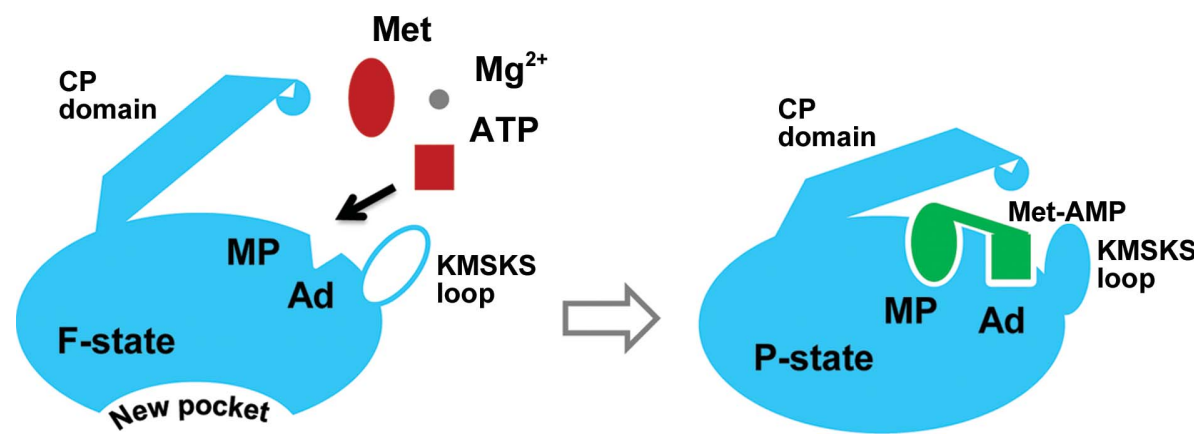

Figure 6

A model of the induced-fit mechanism in ligand binding. Our structural characterization suggests that MtMetRS employs a induced-fit mechanism in ligand binding. In the F-state structure (left) the methionine pocket (MP) does not form and the AMP pocket (Ad) is too narrow to accommodate adenosine. The CP domain is in an open conformation and the KMSKS loop is disordered. In the presence of methionine and ATP, the intermediate product Met-AMP is generated and it accommodates substrate pockets (right). Met-AMP induces the formation of MP and Ad pockets, the architectures of which restore a normal conformation similar to other P-state MtMetRS structures. The CP domain shifts to a closed conformation and the KMSKS loop becomes highly disordered. Owing to the large conformational rearrangements that occur in the F-state structure, F-state MtMetRS exhibits a nonproductive conformation and a new pocket forms on the opposite side to the active site, providing a possible strategy for inhibitor design. Details of the new pocket formed in the F-state structure are shown in Supplementary Fig. S5(e).
2012). The induced-fit mechanism means that the pocket does not pre-exist before ligand binding; thus, the ligand must induce some change in the enzyme structure to form a pocket first. In the case of conformational selection, the pockets are already formed before ligand binding. Different models of ligand recognition have been found in the context of tRNA charging. The bacteria trans-editing enzyme ProXp-ala uses a conformation-selection strategy to discriminate Ala-tRNA ${ }^{\text {Pro }}$ from Pro-tRNA ${ }^{\text {Pro }}$ and thus correct mischarging errors (Danhart et al., 2017). Datt and Sharma questioned the prevailing induced-fit mechanism for ATP recognition by tyrosyl-tRNA synthetases, which involves conformational changes of the KMSKS loop upon ligand binding (Datt \& Sharma, 2014). Based on a PDB-wide structural analysis, they suggested that an extended conformational selection induced-fit mechanism alone.

The induced-fit mechanism employed by MtMetRS may offer an explanation for the slow growth phenomenon of MTB owing to an intrinsic slow protein-synthesis rate. Srivastava and coworkers reported in vitro reconstitution of the protein translation system from purified mycobacterial components (Srivastava et al., 2016). They showed that the in vitro proteinynthesis activity of purified $E$. coli components is significantly might act as a speed-limiting factor for protein synthesis.

MetRSs play a critical role in both bacteria and humans. Therefore, a prerequisite for a potential drug lead is a high selectivity for the pathogen enzyme over the host enzyme (Ochsner et al., 2007). There are two human MetRS: a mitochondrial MetRS belonging to the MetRS1 subfamily and a et al., 2003). Structural comparison of human cytosolic MetRS (HcMetRS; PDB entry 5gl7) and MtMetRS gives a $D A L I$ $Z$-score of 35.8 and an r.m.s.d. value of $1.3 \AA$ with $451 \mathrm{C}^{\alpha}$ atoms aligned. It also shows that HcMetRS and MtMetRS have nearly identical substrate-binding patterns. Interestingly, we found two specific hydrophobic pockets outside the catalytic core of MtMetRS, which might be potential targets for drug design (Supplementary Fig. S5). The inhibitors that bind to either of these pockets might be able to lock MtMetRS in the unbound state and disable MtMetRS activity. This may be a useful target for the design of new drugs targeting $M$. tuberculosis.

In summary, our structural characterization of MtMetRS provides a high-resolution structural framework for drug design. The observed conformational differences between the F-state and P-state MtMetRS structures provide valuable input that is necessary 
for the successful development of inhibitors specifically targeting M. tuberculosis.

\section{Related literature}

The following references are cited in the Supporting Information for this article: Kalogerakos et al. (1980), Kohda et al. (1987), Nureki et al. (1993), Schmitt et al. (1997) and Schwob et al. (1988).

\section{Funding information}

The following funding is acknowledged: National Natural Science Foundation of China (award No. 11775308 and 81401714); Beijing Municipal Natural Science Foundation (award No. 7182117 and 7174288); Chinese Academy of Medical Science (CAMS) Innovation Fund for Medical Sciences (award No. 2017-I2M-1-014); National Key Research and Development Program of China (award No. 2016YFD0500300); Fundamental Research Funds for the Central Universities (award No. 2016ZX310054); Non-profit Central Research Institute Fund of Chinese Academy of Medical Sciences (award No. 2017PT31049).

\section{References}

Adams, P. D. et al. (2010). Acta Cryst. D66, 213-221.

Barros-Álvarez, X., Turley, S., Ranade, R. M., Gillespie, J. R., Duster, N. A., Verlinde, C. L. M. J., Fan, E., Buckner, F. S. \& Hol, W. G. J. (2018). Acta Cryst. F74, 245-254.

Bastos, M. L. et al. (2014). Clin. Infect. Dis. 59, 1364-1374.

Carugo, O. \& Argos, P. (1997). Protein Sci. 6, 2261-2263.

Chetty, S., Ramesh, M., Singh-Pillay, A. \& Soliman, M. E. (2017). Bioorg. Med. Chem. Lett. 27, 370-386.

Crepin, T., Schmitt, E., Blanquet, S. \& Mechulam, Y. (2004). Biochemistry, 43, 2635-2644.

Crepin, T, Schmitt, E., Mechulam, Y., Sampson, P. B., Vaughan, M. D., Honek, J. F. \& Blanquet, S. (2003). J. Mol. Biol. 332, 59-72.

Critchley, I. A., Green, L. S., Young, C. L., Bullard, J. M., Evans, R. J., Price, M., Jarvis, T. C., Guiles, J. W., Janjic, N. \& Ochsner, U. A. (2009). J. Antimicrob. Chemother. 63, 954-963.

Critchley, I. A. \& Ochsner, U. A. (2008). Curr. Opin. Chem. Biol. 12, 409-417.

Critchley, I. A., Young, C. L., Stone, K. C., Ochsner, U. A., Guiles, J., Tarasow, T. \& Janjic, N. (2005). Antimicrob. Agents Chemother. 49, 4247-4252.

Danhart, E. M., Bakhtina, M., Cantara, W. A., Kuzmishin, A. B., Ma, X., Sanford, B. L., Vargas-Rodriguez, O., Košutić, M., Goto, Y., Suga, H., Nakanishi, K., Micura, R., Foster, M. P. \& Musier-Forsyth, K. (2017). Proc. Natl Acad. Sci. USA, 114, E6774E6783.

Datt, M. \& Sharma, A. (2014). J. Struct. Funct. Genomics, 15, 45-61. Deniziak, M. A. \& Barciszewski, J. (2001). Acta Biochim. Pol. 48, 337-350.

Dundas, J., Ouyang, Z., Tseng, J., Binkowski, A., Turpaz, Y. \& Liang, J. (2006). Nucleic Acids Res. 34, W116-W118.

Emsley, P., Lohkamp, B., Scott, W. G. \& Cowtan, K. (2010). Acta Cryst. D66, 486-501.

Gadakh, B. \& Van Aerschot, A. (2012). Expert Opin. Ther. Pat. 22, 1453-1465.

Gentry, D. R., Ingraham, K. A., Stanhope, M. J., Rittenhouse, S., Jarvest, R. L., O'Hanlon, P. J., Brown, J. R. \& Holmes, D. J. (2003). Antimicrob. Agents Chemother. 47, 1784-1789.
Ghosh, G., Brunie, S. \& Schulman, L. H. (1991). J. Biol. Chem. 266, 17136-17141.

Green, L. S., Bullard, J. M., Ribble, W., Dean, F., Ayers, D. F., Ochsner, U. A., Janjic, N. \& Jarvis, T. C. (2009). Antimicrob. Agents Chemother. 53, 86-94.

Holm, L. \& Laakso, L. M. (2016). Nucleic Acids Res. 44, W351W355.

Hong-Min, W. \& Xiao-Hong, Z. (2015). Clin. Infect. Dis. 60, 12851286.

Ibba, M. \& Söll, D. (2000). Annu. Rev. Biochem. 69, 617-650.

Ingvarsson, H., Jones, T. A. \& Unge, T. (2009). Acta Cryst. F65, 618620.

Ingvarsson, H. \& Unge, T. (2010). FEBS J. 277, 3947-3962.

James, B. W., Williams, A. \& Marsh, P. D. (2000). J. Appl. Microbiol. 88, 669-677.

Jørgensen, R., Søgaard, T. M. M., Rossing, A. B., Martensen, P. M. \& Justesen, J. (2000). J. Biol. Chem. 275, 16820-16826.

Kabsch, W. (2010). Acta Cryst. D66, 125-132.

Kalogerakos, T., Dessen, P., Fayat, G. \& Blanquet, S. (1980). Biochemistry, 19, 3712-3723.

Koh, C. Y., Kim, J. E., Shibata, S., Ranade, R. M., Yu, M., Liu, J., Gillespie, J. R., Buckner, F. S., Verlinde, C. L. M. J., Fan, E. \& Hol, W. G. J. (2012). Structure, 20, 1681-1691.

Kohda, D., Yokoyama, S. \& Miyazawa, T. (1987). J. Biol. Chem. 262, 558-563.

Larson, E. T., Kim, J. E., Zucker, F. H., Kelley, A., Mueller, N., Napuli, A. J., Verlinde, C. L. M. J., Fan, E., Buckner, F. S., Van Voorhis, W. C., Merritt, E. A. \& Hol, W. G. J. (2011). Biochimie, 93, 570582.

Li, R., Macnamara, L., Leuchter, J., Alexander, R. \& Cho, S. (2015). Int. J. Mol. Sci. 16, 15872-15902.

Liebschner, D., Afonine, P. V., Moriarty, N. W., Poon, B. K., Sobolev, O. V., Terwilliger, T. C. \& Adams, P. D. (2017). Acta Cryst. D73, 148-157.

Maskell, D. P., Renault, L., Serrao, E., Lesbats, P., Matadeen, R., Hare, S., Lindemann, D., Engelman, A. N., Costa, A. \& Cherepanov, P. (2015). Nature (London), 523, 366-369.

McCoy, A. J. (2007). Acta Cryst. D63, 32-41.

Mechulam, Y., Schmitt, E., Maveyraud, L., Zelwer, C., Nureki, O., Yokoyama, S., Konno, M. \& Blanquet, S. (1999). J. Mol. Biol. 294, 1287-1297.

Nakanishi, K., Ogiso, Y., Nakama, T., Fukai, S. \& Nureki, O. (2005). Nature Struct. Mol. Biol. 12, 931-932.

Nureki, O., Kohno, T., Sakamoto, K., Miyazawa, T. \& Yokoyama, S. (1993). J. Biol. Chem. 268, 15368-15373.

Ochsner, U. A., Bell, S. J., O’Leary, A. L., Hoang, T., Stone, K. C., Young, C. L., Critchley, I. A. \& Janjic, N. (2009). J. Antimicrob. Chemother. 63, 964-971.

Ochsner, U. A., Sun, X., Jarvis, T., Critchley, I. \& Janjic, N. (2007). Expert Opin. Investig. Drugs, 16, 573-593.

Pantoliano, M. W., Petrella, E. C., Kwasnoski, J. D., Lobanov, V. S., Myslik, J., Graf, E., Carver, T., Asel, E., Springer, B. A., Lane, P. \& Salemme, F. R. (2001). J. Biomol. Screen. 6, 429-440.

Schmitt, E., Panvert, M., Mechulam, Y. \& Blanquet, S. (1997). Eur. J. Biochem. 246, 539-547.

Schwob, E., Sanni, A., Fasiolo, F. \& Martin, R. P. (1988). Eur. J. Biochem. 178, 235-242.

Seiradake, E., Harlos, K., Sutton, G., Aricescu, A. R. \& Jones, E. Y. (2010). Nature Struct. Mol. Biol. 17, 398-402.

Serre, L., Verdon, G., Choinowski, T., Hervouet, N., Risler, J. L. \& Zelwer, C. (2001). J. Mol. Biol. 306, 863-876.

Spencer, A. C., Heck, A., Takeuchi, N., Watanabe, K. \& Spremulli, L. L. (2004). Biochemistry, 43, 9743-9754.

Srivastava, A., Asahara, H., Zhang, M., Zhang, W., Liu, H., Cui, S., Jin, Q. \& Chong, S. (2016). PLoS One, 11, e0162020.

Sugiura, I., Nureki, O., Ugaji-Yoshikawa, Y., Kuwabara, S., Shimada, A., Tateno, M., Lorber, B., Giegé, R., Moras, D., Yokoyama, S. \& Konno, M. (2000). Structure, 8, 197-208. 


\section{research papers}

Vogt, A. D. \& Di Cera, E. (2012). Biochemistry, 51, 5894-5902.

Vondenhoff, G. H. M. \& Van Aerschot, A. (2011). Eur. J. Med. Chem. 46, 5227-5236.
Yaginuma, H., Kawai, S., Tabata, K. V., Tomiyama, K., Kakizuka, A., Komatsuzaki, T., Noji, H. \& Imamura, H. (2014). Sci. Rep. 4, 6522 . 